\title{
The challenges of novel contrast agents for the imaging diagnosis of hepatocellular carcinoma
}

\author{
Carmen Ayuso · Jordi Bruix
}

Received: 26 September 2013/ Accepted: 29 November 2013/Published online: 28 December 2013

(C) Asian Pacific Association for the Study of the Liver 2013

The aim of early detection plans in patients at risk of hepatocellular carcinoma (HCC) is to detect the tumor at an evolutionary phase, when a cure with long-term diseasefree survival is highly likely [1]. Accordingly, the goal is to detect and diagnose tumors when they are smaller than $2 \mathrm{~cm}$ in size, the so-called BCLC stage 0 . Beyond this cutoff, the prevalence of satellites and/or microvascular invasion is significantly increased and treatments such as resection, transplant, and ablation will be plagued by recurrence due to dissemination prior to treatment [1]. While this goal is easy to frame, establishing accurate diagnosis in such small nodules is a major clinical challenge. Tumor markers, including alpha-fetoprotein and novel ones such as glypican or PIVKA, have no major usefulness for screening and diagnosis [1], and thus, as in most other cancer types, diagnosis would require a positive biopsy. Nevertheless, biopsy is known to have a suboptimal sensitivity (around 60-70 \%) [1, 2]. It provides a limited amount of tissue, and the fact that such small HCCs are usually well differentiated makes it difficult to confidently distinguish HCC from dysplastic or regenerative nodules. Even expert pathologists would not have a $100 \%$ agreement without false positives and false negatives in such small malignant nodules. Immunohistochemical staining for glypican 3, heat-shock protein 90, or survivin may reinforce HCC suspicion, but they do not increase the diagnostic rate. Diagnostic signatures based on gene expression have also been proposed but have not been validated, and such molecular diagnosis is not yet in

C. Ayuso $\cdot$ J. Bruix $(\bowtie)$

BCLC Group, Hepatic Oncology, Hospital Clínic, CIBEREHD, IDIBAPS, University of Barcelona, Villarroel 170,

08036 Barcelona, Spain

e-mail: jbruix@clinic.ub.es clinical practice. Even if biopsy would have $100 \%$ sensitivity, it would not be feasible in some patients because of the location or the presence of clotting disorders.

All these comments explain the development of imaging criteria for the non-invasive diagnosis of HCC [1]. The increased arterial blood supply that characterizes HCC provides the basis for the increased contrast uptake in the arterial phase of dynamic techniques, such as computed tomography and magnetic resonance. This is a hallmark of malignancy and, if it is followed by hypointensity in the venous/delayed phases, the profile is specific for HCC [2]. This hypointense appearance of the hypervascular focal lesions known as "wash-out" is due to the venous supply being less prominent in HCC as compared to the surrounding liver, and, thus, the arrival of contrast through the portal vein, so that the liver intensity is enhanced as compared to the tumor. Several studies have validated such a profile as almost $100 \%$ specific for HCC [2-4]. Interestingly, while intrahepatic cholangiocarcinoma may display increased arterial blood supply, it does not show washout and this is key for the distinction between these two entities, which have a sharply different prognosis and treatment $[5,6]$. It is important to note that ICC incidence is on the rise and cirrhosis is a major risk for it. Thus, differentiation between HCC and ICC is not futile. Nevertheless, as happens with biopsy, the diagnostic sensitivity of CT or MR for small HCC $(<2 \mathrm{~cm})$ is far from $100 \%$. Increased arterial blood supply appears along tumor growth and, currently, just $60-70 \%$ of HCC $<2 \mathrm{~cm}$ can be confidently diagnosed by imaging techniques [2].

All these limitations explain the need to further improve the diagnostic accuracy of imaging techniques in order to set a non-invasive diagnosis avoiding the request for a positive biopsy. The use of organ-specific contrast agents such as gadoxetic acid (GA) aims to fulfill this goal. GA is 
a combined hepatobiliary (HB) and Gadolinium-chelates contrast agent, suitable for regular dynamic multiphasic studies, that offers the possibility to obtain both the conventional arterial and venous phases, but also a 20 -min delayed HB phase. At this delayed time point, the hepatocytes have taken up the $50 \%$ of the administered dose [7-10]. Therefore, non-hepatocyte focal lesions and those with non-functional hepatocytes, such as most HCCs, should not accumulate contrast. This results in low signal intensity foci relative to the intensity of the liver parenchyma in the HB phase of GA-enhanced MR. While this background would make GA-MRI an optimal technique, there are a number of issues that need active research.

Several studies suggest a potential diagnostic value [11-15], but no robust prospective study has defined its added diagnostic value in the clinical setting. Very early HCC with well-preserved hepatocyte function may show GA uptake and offer false negative results. In addition, as previously mentioned, ICC may also emerge in a cirrhotic liver, and likely this opens a potential for false positive diagnosis. Finally, the technicalities for the optimal use of GA for an adequate reading of the arterial and venous phases of MRI as done currently with gadolinium chelates are not well established. The injection rate of the small amount of contrast injected for GA-MRI may limit the proper recognition of the contrast uptake in the arterial phase and, thus, impair the use of the already validated criteria based on the recognition of arterial uptake followed by wash-out. Even wash-out recognition in such nodules may be impaired, as uptake of GA in the biliary phase may overlap with the delayed venous one. Accordingly, the injection of higher volumes or the use of different injection rates have been proposed to optimize the information offered by GA-MR.

Prior studies have evaluated the impact of injection rate and the amount of contrast injected with controversial results. Zech et al. [16] compared in an experimental study the influence of regular to double doses and different rates of injection (1-2 mL/s) on the enhancement of liver vasculature in GA-MR and Gd-DTPA. They found that the arterial enhancement in GA-MR injected at $1 \mathrm{~mL} / \mathrm{s}$ was comparable to Gd-DTPA. Haradome et al. [17] reported in a retrospective study on 216 patients that fluoroscopic triggering GA-MR and slow rate injection provide proper arterial phase images. Schmid-Tannwald et al. [18] compared two injection rates (1 and $2 \mathrm{~mL} / \mathrm{s})$ for GA-MR dynamic studies in 80 patients and reported higher enhancement in the aorta in the arterial phase with the lower injection rate. Feuerlein et al. [19] compared the enhancement characteristics of liver parenchyma, portal vein, and the portal vein to liver contrast with two HB contrast agents in 70 patients. The authors found that contrast in the arterial and portal venous phases was lower when GA was used compared to Gd-BOPTA.

It is important to note that such studies do not include patients with liver tumors. Such information is the focus of the manuscript by Chen et al. [20] published in this issue of Hepatology International. The authors describe that, in hypervascular HCCs, the enhancement in the arterial and portal phases is lower than that observed when using the conventional non-specific gadolinium. The authors found that the relative contrast difference between the liver and HCC was significantly less when using GA as compared with a nonspecific gadolinium, but, interestingly, it was increased in the portal phase. This resulted in a better definition of the hypointense lesions that may be due to the overlap of the delayed venous phase and the early HB uptake of GA by the nontumoral liver.

In summary, all studies suggest that GA-MR may become a relevant asset for the diagnosis and staging of patients with suspected or proven HCC, but major clinical research is needed to clarify how it should be used and what kind of information is going to be key for clinical decision making. Thereby, while some of the issues previously depicted may hamper a major contribution of GAMR in the diagnostic area, it may well be that it becomes relevant in the evaluation of tumor burden. Disseminated intrahepatic nests are formed by less differentiated hepatocytes and, as such, they will probably appear as hypointense in the HB phase. As a consequence, HCC staging prior to treatment indication would be improved and would optimize the use of the therapeutic options. If such expectation is proven, GA-MR will become an instrumental technique for the proper management of patients diagnosed with HCC.

\section{References}

1. Bruix J, Sherman M. Management of hepatocellular carcinoma: an update. Hepatology 2011;53:1020-1022

2. Forner A, Vilana R, Ayuso C, et al. Diagnosis of hepatic nodules $20 \mathrm{~mm}$ or smaller in cirrhosis: prospective validation of the noninvasive diagnostic criteria for hepatocellular carcinoma. Hepatology 2008;47:97-104

3. Sangiovanni A, Manini MA, Iavarone M, et al. The diagnostic and economic impact of contrast imaging techniques in the diagnosis of small hepatocellular carcinoma in cirrhosis. Gut 2010;59:638-644

4. Rimola J, Forner A, Tremosini S, et al. Non-invasive diagnosis of hepatocellular carcinoma $\leq 2 \mathrm{~cm}$ in cirrhosis. Diagnostic accuracy assessing fat, capsule and signal intensity at dynamic MRI. J Hepatol 2012;56:1317-1323

5. Rimola J, Forner A, Reig M, et al. Cholangiocarcinoma in cirrhosis: absence of contrast washout in delayed phases by magnetic resonance imaging avoids misdiagnosis of hepatocellular carcinoma. Hepatology 2009;50:791-798 
6. Iavarone M, Piscaglia F, Vavassori S, et al. Contrast enhanced CT-scan to diagnose intrahepatic cholangiocarcinoma in patients with cirrhosis. J Hepatol 2013;58:1188-1193

7. Schuhmann-Giampieri G, Schmitt-Willich H, Press WR, et al. Preclinical evaluation of Gd-EOB-DTPA as a contrast agent in MR imaging of the hepatobiliary system. Radiology 1992;183:59-64

8. Vogl TJ, Kümmel S, Hammerstingl R, et al. Liver tumors: comparison of MR imaging with Gd-EOB-DTPA and Gd-DTPA. Radiology 1996;200:59-67

9. Frericks B, Loddenkemper C, Huppertz A, et al. Qualitative and quantitative evaluation of hepatocellular carcinoma and cirrhotic liver enhancement using Gd-EOB-DTPA. AJR 2009;193:1053-1060

10. Ayuso C, Rimola J, García-Criado A. Imaging of HCC. Abdom Imaging 2012;37:215-230

11. Golfieri R, Renzulli M, Lucidi V, et al. Contribution of the hepatobiliary phase of Gd-EOB-DTPA-enhanced MRI to Dynamic MRI in the detection of hypovascular small $(\leq 2 \mathrm{~cm}) \mathrm{HCC}$ in cirrhosis. Eur Radiol 2011;21:1233-1242

12. Granito A, Galassi M, Piscaglia F, et al. Impact of gadoxetic acid (Gd-EOB-DTPA)-enhanced magnetic resonance on the noninvasive diagnosis of small hepatocellular carcinoma: a prospective study. Aliment Pharmacol Ther 2013;37:355-363

13. Golfieri R, Grazioli L, Orlando E, et al. Which is the best MRI marker of malignancy for atypical cirrhotic nodules: hypointensity in hepatobiliary phase alone or combined with other features? Classification after Gd-EOB-DTPA administration. J Magn Reson Imaging 2012;36:648-657

14. Bartolozzi C, Battaglia V, Bargellini I, et al. Contrast-enhanced magnetic resonance imaging of 102 nodules in cirrhosis: correlation with histological findings on explanted livers. Abdom Imaging 2013;38:290-296

15. Bashir MR, Gupta RT, Davenport MS, et al. Hepatocellular carcinoma in a North American population: does hepatobiliary MR imaging with Gd-EOB-DTPA improve sensitivity and confidence for diagnosis? J Magn Reson Imaging 2013;37:398-406

16. Zech CJ, Vos B, Nordell A, et al. Vascular enhancement in early dynamic liver MR imaging in an animal model: comparison of two injection regimen and two different doses Gd-EOB-DTPA (gadoxetic acid) with standard Gd-DTPA. Invest Radiol 2009; 44:305-310

17. Haradome H, Grazioli L, Tsunoo M, et al. Can MR fluoroscopic triggering technique and slow rate injection provide appropriate arterial phase images with reducing artifacts on gadoxetic acidDTPA (Gd-EOB-DTPA)-enhanced hepatic MR imaging? J Magn Reson Imaging 2010;32:334-340

18. Schmid-Tannwald C, Herrmann K, Oto A, et al. Optimization of the dynamic, Gd-EOB-DTPA-enhanced MRI of the liver: the effect of the injection rate. Acta Radiol 2012;53:961-965

19. Feuerlein S, Gupta RT, Boll DT, Merkle EM. Hepatocellular MR contrast agents: enhancement characteristics of liver parenchyma and portal vein after administration of gadoxetic acid in comparison to gadobenate dimeglumine. Eur J Radiol 2012;81: 2037-2041

20. Chen CZ, Rao SHS-X, Ding Y, et al. Hepatocellular carcinoma $20 \mathrm{~mm}$ or smaller in cirrhosis patients: early magnetic resonance enhancement by gadoxetic acid compared with gadopentetate dimeglumine. Hepatol Int 2013;. doi:10.1007/ s12072-013-9467-7 